\title{
Is there any place for immune-checkpoint inhibitors in the treatment algorithm of fusion-driven non-small cell lung cancer? - a literature review
}

\author{
Gianmarco Leone, Francesco Passiglia, Paolo Bironzo, Valentina Bertaglia, Silvia Novello \\ Department of Oncology, University of Turin, S. Luigi Gonzaga Hospital, Orbassano, Italy \\ Contributions: (I) Conception and design: F Passiglia, S Novello; (II) Administrative support: G Leone; (III) Provision of study materials or patients: \\ G Leone, F Passiglia; (IV) Collection and assembly of data: G Leone, F Passiglia; (V) Data analysis and interpretation: F Passiglia, P Bironzo, V \\ Bertaglia; (VI) Manuscript writing: All authors; (VII) Final approval of manuscript: All authors. \\ Correspondence to: Francesco Passiglia, MD. Department of Oncology, University of Turin, S. Luigi Gonzaga Hospital, Regione Gonzole, 10,10034 \\ Orbassano (TO), Italy. Email: francesco.passiglia@unito.it.
}

\begin{abstract}
The advent of immune-checkpoint inhibitors (ICIs) targeting the programmed death-1 (PD1)/programmed death ligand-1 (PD-L1) axis, produced a paradigm change of the treatment algorithm for metastatic, non-oncogene addicted, non-small cell lung cancer (NSCLC). However, the majority of patients with oncogene-addicted disease have been excluded from the "immunotherapy revolution", thus the clinical efficacy of these agents in this subset of patients remains largely unknown. Although pre-clinical evidence provided a good rationale to pursue the investigation of ICI treatment in specific subgroups of oncogeneaddicted NSCLC, current available evidence suggested that tumors harboring molecular alterations likely do not represent the best candidate to single agent ICI therapy. Furthermore, the prospect of further improving overall survival (OS) with the combination of tyrosine kinase inhibitors (TKIs) and ICIs led to unexpected poor results and safety issues in recent phase I trials exploring different therapeutic associations. Conversely, the combination of immunotherapy and chemotherapy is emerging as a potential effective strategy in specific subsets of NSCLC patients harboring oncogenic drivers. In this review we particularly focus on the subgroup of patients whose disease harbor oncogenic rearrangements, summarizing current evidence from preclinical and clinical studies and discussing their practical implications, in order to define the potential role of ICIs in the clinical management of fusion-driven NSCLC.
\end{abstract}

Keywords: Immune-checkpoint inhibitors (ICIs); programmed death-1/programmed death ligand-1 (PD-1/PDL1); rearrangements; fusion; non-small cell lung cancer (NSCLC)

Submitted Jun 01, 2020. Accepted for publication Aug 10, 2020.

doi: $10.21037 /$ tlcr-20-710

View this article at: http://dx.doi.org/10.21037/tlcr-20-710

\section{Introduction}

The advent of targeted therapies and immune checkpoint inhibitors (ICIs) has radically changed the therapeutic landscape of non-small cell lung cancer (NSCLC) over the last years, certainly contributing to the increase of 5 -year survival rate, reported to be $21.7 \%$ nowadays, compared to $17.2 \%$ about a decade ago (1). Although the efficacy of tyrosine kinase inhibitors (TKIs) is limited to a subset of NSCLC patients harboring specific oncogenic drivers, their implementation in the clinical practice dramatically improved patients' survival and quality of life in stage IV diseases (2), delaying the use of less tolerated chemotherapeutic agents. Alongside epidermal growth factor receptor $(E G F R)$, v-Raf murine sarcoma viral oncogene homolog B $(B R A F)$, Kirsten rat sarcoma 2 viral oncogene homolog (KRAS) and c-MET-exon 14 mutations (hepatocyte growth factor receptor), oncogenic fusions involving anaplastic lymphoma kinase $(A L K), \mathrm{v}$-ROS avian 
UR2 sarcoma virus oncogene homolog 1 (ROS1), rearranged during transfection (RET), and tropomyosin receptor kinase A (NTRK) genes represent the most successfully druggable targets in NSCLC, with new-generation TKIs already available or coming soon in the clinical setting. This means to progressively extend the number of potential candidates to personalized treatment strategies in the upcoming years. Conversely, the majority of patients with oncogene-addicted NSCLC have been excluded from the "immunotherapy revolution", due to the anticipated poor response from preclinical data (3) and the lack of activity observed in the small group of EGFR-positive patients included in the second-line ICIs clinical studies (4). As a consequence, the clinical development of ICIs, both as single agent and in combination with first-line platinum-based chemotherapy has been exclusively limited to the EGFR/ALK wild-type NSCLC population. In absence of prospective data on immune-chemotherapy combinations in oncogene-addicted NSCLC, a subgroup analysis of the phase III IMpower-150 trial showed that the survival benefit derived from the addition of atezolizumab to the bevacizumab-chemotherapy combination regimen in advanced non-squamous NSCLC, was extended also to the EGFR-mutant population [hazard ratio (HR): 0.60; 95\% confidence intervals (CIs): 0.31-1.14] (5). This evidence has driven the regulatory approval of this treatment regimen after EGFR-TKI failure, but the low number of evaluated patients and the lack of detailed information regarding tumor molecular alterations and previous TKI lines reduced the scientific reliability and the clinical value of such data. Conversely, the prospect of further improving overall survival (OS) with the combination of TKIs and ICIs lead to unexpected poor results and safety issues in recent phase I trials exploring different drug associations. Based on current available clinical evidence, immunotherapy and TKIs are emerging as two different therapeutic approaches with clinical efficacy limited to two distinct, separate subsets of NSCLC patients. However, preclinical studies and translational data revealed interesting dynamic molecular networks between tumor cells intrinsic oncogenic signaling and immune microenvironment, with potential influence on immunomodulating process, which provide a strong rationale to pursue the investigation of ICI treatment efficacy in particular subsets of oncogene-addicted NSCLC. This review will particularly focus on the subgroup of patients whose disease harbors oncogenic rearrangements, summarizing current evidence from preclinical and clinical studies and discussing their practical implications, in order to define the potential role of ICIs therapy in the clinical management of fusion-driven NSCLC. We present the following article in accordance with the NARRATIVE REVIEW reporting checklist (available at http://dx.doi. org/10.21037/tlcr-20-710).

\section{Search strategy}

The literature search was conducted using PubMed database. For each rearrangement analyzed in this review the following terms were used: "Rearrangement' and PDL1 expression", "Rearrangement' and immunotherapy", "Rearrangement' and checkpoint inhibitors" and "NSCLC Fusions" (e.g., "ALK and checkpoint inhibitors"). Relevant studies published between January 2015 and May 2020 were selected. Literature citations within selected studies were also searched to find other potentially relevant studies.

\section{ALK}

The introduction of second-generation ALK inhibitors into clinical practice has dramatically improved the survival outcomes of ALK-positive NSCLC, with unmatched results even compared to other oncogene-addicted NSCLC. Alectinib and brigatinib have largely replaced crizotinib as first line treatment in ALK-positive patients with advanced disease, based on the results of the phase III ALEX (6) and ALTA-1L (7) trial, respectively. Lorlatinib is the first third-generation ALK inhibitor that has been approved as a second line treatment after ALK-TKI progression in this subgroup of patients, based on the B7461001 phase II trial results (8). Nowadays, the treatment algorithm for advanced ALK-positive NSCLC should include a second-generation TKIs (alectinib or brigatinib preferred over ceritinib) in first line therapy, eventually followed by lorlatinib or platinumbased chemotherapy at disease progression. Immunotherapy represent a potential option in heavily pre-treated patients who maintain a good performance status.

\section{Pre-clinical background}

Several studies illustrated the correlation between ALKrearrangements and programmed death ligand-1 (PDL1) expression. PD-L $1 \mathrm{mRNA}$ and surface protein expression are significantly higher in EML4-ALK-positive lines compared to both EGFR and ALK wild-type cells. Moreover, wild-type cells transfected with an EML4-ALK expression vector showed an increase of PD-L1 levels, while alectinib downregulated PD-L1 expression in EML4-ALK- 
positive models (9). PD-L1 TPS score usually increased in repeated biopsy after ALK-TKIs therapies, reaching about $57 \%$ in heavily pre-treated samples (10).

It has been largely demonstrated that ALKrearrangements upregulates PD-L1 expression via STAT3 signaling in both anaplastic large cell lymphoma (11) and lung cancer (12). Indeed, PD-L1 expression significantly decreased in EML4-ALK-positive cell lines when treated with STAT3 inhibitors. Data from protein-DNA binding assays suggest that STAT3 exerts its activity by directly binding to PD-L1 promoter region. Marzec et al. used CRISPR/Cas9 library screening to find other nuclear proteins (GRB2/SOS1, IRF4, BATF3) that play a crucial role for ALK-mediated PD-L1 expression in NPM-ALKpositive anaplastic large-cell lymphoma (11). Indeed, these transcription factors seem to be equally involved for the PDL1 upregulation in EML4-ALK-positive adenocarcinomas (ADCs). HIF- $1 \alpha$ cooperates with STAT3 to increase PDL1 levels under hypoxia. While HIF-1 $\alpha$ is known to act as a transcription factor for PD-L1 in different tumor types (13), its effect seems to be amplified in EML4-ALK-positive lung cancer. The interaction between STAT3 and HIF-1 $\alpha$ might be critical to enhance PD-L1 expression in EML4-ALKpositive cell lines (12). Recently, Nouri et al. identified ALK as a critical regulator of the Hyppo pathway, which seems to be involved in both tumorigenesis and immune evasion processes (14). The interaction between EML4-ALK and the yes-associated protein 1/transcriptional co-activator with PDZ binding motif (YAP/TAZ) complex resulted in a STAT3 independent PD-L1 upregulation (14).

Correlations between ALK-rearrangements and other known immune-related biomarkers, such as CD8+ $\mathrm{T}$ cell infiltration, HLA-class I receptor expression and total mutation burden (TMB), have been also investigated. The combination of PD-L1 expression on tumor or stromal cells and intratumoral infiltration by $\mathrm{PD}-1^{+} \mathrm{CD} 8^{+} \mathrm{T}$ cells tended to be more frequent in ALK-positive than in nonALK-rearranged ADCs. In addition, high HLA-class I expression on tumor cells with intratumoral infiltration by $\mathrm{PD}-1^{+} / \mathrm{CD} 8^{+} \mathrm{T}$ cells appeared to be more frequent in ALKpositive ADCs (15). Interestingly, a positive correlation between intratumoral infiltration by $\mathrm{CD} 8^{+} \mathrm{T}$ cells and ALK-rearrangements was not constantly observed in other tumors (16). Conversely, TMB level was significantly lower (mean 3.1 mutation/Mb) in ALK-positive lung cancer compared to that observed in unselected NSCLC patients (17). Although a prognostic role of these biomarkers in ALK-rearranged NSCLC has not been demonstrated yet (15), in a large retrospective database including 715 EGFR/ALK-positive lung cancer samples, with only $10 \%$ reporting ALK-rearrangement, the detection of $\mathrm{PD}-\mathrm{Ll}^{+} /$ $\mathrm{CD}^{+}$cells in the tumor microenvironment was associated to worse OS (44.3 months compared with 93.4 months in $\mathrm{PD}-\mathrm{L}^{-} / \mathrm{CD}^{-}$group) (18). Finally, pre-clinical data showed that the therapeutic inhibition of ALK oncogenic signaling increased $\mathrm{T}$-cells interactions, proliferation and tumor infiltration, as well as inflammatory cytokine release, thus providing biological rationale for immune-target combination strategies (19).

\section{Clinical evidence}

Despite the theoretically favorable preclinical background, available evidence from different trials testing ICIs for ALK-rearranged ADCs have so far been disappointing. ICIs have been investigated either alone (prospectively and retrospectively) or in combination with chemotherapy or ALK-TKIs (Table 1).

ALK-positive ADCs, as well as other oncogene addicted NSCLC, were poorly represented in the majority of prospective clinical trials investigating single agent ICI both in second and first-line setting (20-22). The ATLANTIC study was a single arm trial investigating durvalumab in NSCLC patients who received at least two previous lines of treatment. In the Cohort 1, 111 patients with ALK and/ or EGFR positive NSCLC were enrolled, including 16 patients with ALK-positive ADCs. Exclusive analysis for OS in the ALK-positive subgroup was not performed, but an exploratory post-hoc analysis showed that all objective responses in the cohort 1 occurred in EGFR+ patients (9\%; 10 out of 111) (23). Only five EML4-ALK-positive patients were included in the OAK trial, comparing atezolizumab $v s$. docetaxel in previously treated NSCLC, while about $50 \%$ of the entire population was untested for ALK-rearrangements (24). Thus, any evaluation about the efficacy of atezolizumab in this subgroup is meaningless. The Impower-150 investigated the association between atezolizumab and bevacizumab with standard platinumbased chemotherapy in non-squamous NSCLC. Thirty-four patients with EML4-ALK-positive disease, experiencing disease progression to at least one previous treatment, were enrolled in the trial (13 in the experimental arm). In EGFR/ ALK-positive population progression-free survival (PFS) and OS were longer within the chemo-immunotherapy combination compared to control arm (PFS: median, $8.3 \mathrm{vs}$. 6.8 months; HR, $0.61 ; 95 \%$ CI, 0.52 to 0.72 ; OS: median, 
Table 1 ICIs activity and safety in fusion-driven NSCLC patients

\begin{tabular}{|c|c|c|c|c|}
\hline Authors [year] & N. patients & Treatment & ORR & AEs grade $\geq 3$ \\
\hline Mazieres et al. [2019] & 23 & PD-1/PD-L1 inhibitors & $0 \%$ & NR \\
\hline $\mathrm{Ng}$ et al. [2019] & 13 & PD-1/PD-L1 inhibitors & $0 \%$ & NR \\
\hline Gainor et al. [2016] & 6 & PD-1/PD-L1 inhibitors & $0 \%$ & NR \\
\hline Bylicki et al. [2020] & 8 & PD-1/PD-L1 inhibitors & $25 \%$ & $8 \%$ ** \\
\hline Chalmers et al. [2019] & 3 & Crizotinib-ipilimumab & $N R^{*}$ & $33 \%$ \\
\hline Spigel et al. [2018] & 13 & Crizotinib-nivolumab & $38 \% *$ & $38 \%$ \\
\hline Patel et al. [2020] & 9 & Crizotinib-pembrolizumab & $55.6 \%$ & $44 \%$ \\
\hline Kim et al. [2018] & 21 & Alectinib-atezolizumab & $85.7 \%$ & $57 \%$ \\
\hline Shaw et al. [2018] & 28 & Lorlatinib-avelumab & $46.4 \%$ & $53.6 \%$ \\
\hline \multicolumn{5}{|l|}{ ROS1 } \\
\hline Mazieres et al. [2019] & 7 & PD-1/PD-L1 inhibitors & $17 \%$ & NR \\
\hline Park et al. [2018] & 12 & CTLA4/PD-1/PD-L1 inhibitors & $25 \%$ & NR \\
\hline \multicolumn{5}{|l|}{$R E T$} \\
\hline Offin et al. [2019] & 16 & CTLA4/PD-1/PD-L1 inhibitors & $0 \%$ & NR \\
\hline Mazieres et al. [2019] & 16 & PD-1/PD-L1 inhibitors & $6 \%$ & NR \\
\hline
\end{tabular}

*, treatment discontinued due to safety issues; **, overall population enrolled in the trial, included but not limited to ALK/RET positive NSCLC. ICI, immune-checkpoint inhibitor; NSCLC, non-small cell lung cancer; N., number; ORR, objective response rate; AEs, adverse events; PD-1, programmed death-1; PD-L1, programmed death ligand-1; CTLA-4, cytotoxic T-lymphocyte antigen 4; NR, not reported.

9.7 vs. 6.1 months; HR, 0.59; 95\% CI, 0.37 to 0.94$)(25)$. Considering the low number of included patients and the lack of efficacy analysis specifically devoted to ALK-positive subgroup, the exact efficacy of this combination in TKIpre-treated patients with ALK-rearrangements remains currently controversial. A phase II prospective study is currently evaluating the efficacy of pembrolizumab in combination with platinum-based doublet chemotherapy in patients with ALK-rearranged NSCLC with progressive disease following prior TKIs (NCT03242915).
Additional data were available from retrospective studies and small case series. Among 551 pre-treated patients with advanced NSCLC included in the IMMUNOTARGET registry, 23 harbored ALK-rearrangements. All patients received a programmed death-1 (PD-1) or PD-L1 inhibitor at some point of their disease history, especially as a secondor third-line treatment (67\%), and objective responses were not observed in anyone of the ALK-positive patients (26). These results are consistent with another retrospective series of 13 ALK-positive patients collected by $\mathrm{Ng}$ et al., 
where no response were achieved (27). Similar findings from other small series were reported, with overall response rate (ORR) to $\mathrm{PD}-1$ blockade overall ranging from $0 \%$ to $25 \%$ in the ALK-rearranged population (28-30).

Despite a strong biological rationale and the high expectations regarding the association between TKI and immunotherapy, phase I clinical trials disappointing results discouraged to pursue this treatment strategy. In a phase 1 trial the CTLA4 inhibitor ipilimumab was studied in combination with erlotinib or crizotinib in EGFR and ALK-positive NSCLC, respectively. Both treatment arms were associated to unacceptable toxicity, with two of three ALK-positive patients developing hypophysitis and grade 2 pneumonia, respectively (31). Combinations of TKIs and PD1/PDL1 inhibitors were also characterized by high grade adverse events. CheckMate 370 was a 5 -cohort, openlabel phase $1 / 2$ study investigating nivolumab in different settings. Within the cohort E, nivolumab (240 mg every 2 weeks) was associated with crizotinib ( $250 \mathrm{mg}$ twice daily) as a first line therapy for advanced ALK-positive NSCLC. Among the 13 patients enrolled in the study, 5 (38\%) developed severe hepatic toxicities, including two grade 5 toxicities. Hepatic adverse events were reported to be less common with single agent nivolumab ( $0.3 \%$ to $1.5 \%)$ $(20,32)$ and crizotinib (2.3\%) (33), suggesting an additive and synergistic toxic effect for the combination. Enrolment was closed and treatment discontinued in all other patients included in this cohort (34). Another trial investigating the association of pembrolizumab and crizotinib was terminated early due to the very slow accrual. Elevations in transaminase levels was the most common adverse event, but bilirubin increase or hepatic failure was not observed in this study, as toxicities were reversible with pembrolizumab discontinuation (35). Ceritinib and nivolumab combination was evaluated in an open-label, phase $1 \mathrm{~B}$ study. The trial enrolled 16 TKI-naïve and 20 pre-treated ALK-positive patients to receive nivolumab ( $3 \mathrm{mg} / \mathrm{kg}$ every 2 weeks) plus ceritinib (three different dose levels, administrated once daily with a low-fat meal). Despite the study planned a maximum dose of $600 \mathrm{mg} /$ day for ceritinib, all patients received $300 \mathrm{mg} / \mathrm{die}(\mathrm{n}=22)$ or $450 \mathrm{mg} / \mathrm{die}(\mathrm{n}=14)$. Once again, a high rate $(83 \%)$ of grade $\geq 3$ adverse events was reported, with increase in AST/ALT or lipase levels, and rash being the most common. ORR and PFS were $69 \%$ and 16.6 months in the TKI-naïve and 35\% and 4.6 months in the pre-treated subgroups, respectively (36). Although the combination showed some level of activity, particularly in presence of high PD-L1 expression, overall results were comparable to those obtained with ceritinib monotherapy in the ASCEND-4 and ASCEND-5 trials, with the drawback of augmented toxicities $(37,38)$. In another phase I trial, induction therapy with alectinib at the standard dose of $600 \mathrm{mg}$ BID for a week was followed by the association of alectinib (600 mg BID) and atezolizumab (1,200 mg every 3 weeks) (39). Twenty-one ALK-positive patients who had not received any previous treatment were enrolled in the study. Since the median follow-up at the time of data cut off was just 13 months and considering the impressive outcome in terms of PFS reported with alectinib monotherapy in the ALEX trial (40), any assumption about the activity of this combination remains speculative. Conversely, the tolerability profile of the association was really poor, compared to single agent TKI, with 14 patients (67\%) experiencing alectinib dose interruptions or modifications due to grade 3 adverse events (57.1\% treatment-related, mainly rash and liver toxicity). Lastly, Javelin Lung 101 trial evaluated the combination of avelumab with crizotinib or lorlatinib in two different cohorts. The combination of avelumab and crizotinib was investigated in the ALK wild-type population. The avelumab-lorlatinib cohort included 28 ALK-positive patients, reaching an ORR of $46.4 \%$, which could be considered a promising result in a population with unfavorable prognostic factors $36 \%$ had untreated brain metastasis, $71.4 \%$ received two or more previous TKIs) (41). The safety was again the main issue with grade $\geq 3$ adverse events occurring in $53.6 \%$ of cases.

\section{ROS1}

ROS1 tyrosine kinase domains present high affinity with the ALK ones, therefore TKIs targeting ALK-rearrangements, crizotinib and lorlatinib, showed great levels of activity also in ROS1 positive patients $(42,43)$. Entrectinib is a novel TKI that inhibits both ROS1 and NTRK fusion products, showing great efficacy in a heterogeneous group of ROS1 positive NSCLC included in an integrative analysis of three phase I/II trials. (44). Promising results were reported in early-phase clinical trials for repotrectinib and DS-6051b $(45,46)$. Since crizotinib represents the current first-line standard of care in this subgroup of patients, single agent immunotherapy could be considered in patients progressed to previous TKI and chemotherapy.

\section{Pre-clinical background}

The correlation between ROS1 rearrangements and PD- 
L1 expression or immunotherapy response is the least investigated so far among the druggable translocations in NSCLC. While ROS1 and ALK present high homology in their kinase domains, suggesting a similar effect on PD-L1 expression, there are current no preclinical data confirming this hypothesis. ROS1 positive tumors were poorly represented even in retrospective case series $(27,47,48)$, preventing any conclusion about PD-L1 expression in this subgroup of patients. Importantly, ROS1 positive NSCLC shares with ALK- and RET- positive counterparts a lower TMB compared to wild type or EGFR-mutated adenocarcinomas (49).

\section{Clinical evidence}

Seven ROS1 positive NSCLC patients were enrolled within the IMMUNOTARGET cohort. ORR was $17 \%$, while PFS and OS data were not available (26). In a retrospective Korean study 12 ROS1 positive NSCLC patients (among $103,67 \%$ stage IV) received ICIs at some point of their treatment history. ORR was $25 \%$ and PFS ranged from 1.1 to 10.7 months (50) (Table 1). The presence of ROS1 positive NSCLC in prospective phase III trials investigating ICIs both as single agent and as combination with chemotherapy was not assessed, while phase I trials investigating crizotinib/lorlatinib and ICIs combinations were limited to ALK-positive patients.

\section{RET}

RET-rearranged NSCLC is getting more and more interest among clinicians with the upcoming advent of new selective RET inhibitors. Thus, searching for RET fusions is becoming more common in clinical practice alongside EGFR and BRAF mutations and ALK and ROS1 rearrangements. Pralsetinib (BLU-667) and selpercatinib (LOXO-292), are the two major compounds currently under clinical development, with promising activity emerging from the ARROW (51) and the LIBRETTO-001 (52) phase 1 trials, respectively. A phase III trial comparing selpercatinib to platinum-based chemotherapy with or without pembrolizumab as a first line treatment in RET positive NSCLC is currently ongoing (LIBRETTO-431, NCT04194944). Where clinical trials for selective RET inhibitors are not available, chemotherapy and/or immunotherapy remains the current standard first line treatment for RET positive NSCLC.

\section{Pre-clinical background}

As a recent molecular alteration for NSCLC, potential correlation between RET and PD-L1 expression has not been investigated yet on molecular basis. However, RET signaling pathway includes STAT family members (STAT1/3/5) (53), which are known to increase PDL1 expression, as already stated above. In addition, data from studies on multiple endocrine neoplasia type 2 showed that RET positive papillary thyroid carcinoma display a significant immune infiltrate in the tumor microenvironment (54). Whether these results are applicable also to NSCLC remains uncertain. RET positive NSCLC presented significant PD-L1 expression in different case series (49,55-57), with PD-L1 positive (TPS $\geq 1 \%$ ) RET-rearranged tumors accounting for $40-50 \%$ of all cases. Conversely, median TMB was significantly lower in RET positive as compared to wild type NSCLC $(49,56)$, but similar to that reported for ALK and ROS1 positive NSCLC (49).

\section{Clinical evidence}

Screening for RET rearrangements was not required in the main prospective NSCLC trials with single agent ICIs or chemo-immunotherapy combinations, thus the number of RET positive cases enrolled, if any, as well as potential ICI efficacy in this subgroup of enrolled patients, remains currently unknown. Several retrospective series reported generally poor response to ICIs. Offin et al. collected 74 RET positive NSCLC patients, with 16 of them receiving PD-1/PD-L1 inhibitors at some point of their treatment history. No objective response was observed among the 13 patients assessed for clinical and/or radiological response and overall PFS was 3.4 months (56). These results are consistent with the RET cohort included within the IMMUNOTARGET registry, where only one of 16 evaluated patients achieved PR, with median PFS reaching 2.1 months (26). In another retrospective case series, two RET positive patients (out of 14 total cases) with PD-L1 TPS $>50 \%$ received pembrolizumab as first line therapy. PD was observed for both patients after 1 and 2 months respectively (58). Baglivo et al. reported two cases of RET positive NSCLC with PD-L1 expression $>50 \%$ who experienced hyper-progression under Pembrolizumab first line therapy, suggesting that knowing RET status at baseline could be crucial to exclude these patients from immunotherapy treatment (59). More encouraging 
results were obtained by Guisier et al. in a small subgroup of nine RET-positive NSCLC patients who received pembrolizumab or nivolumab $(89 \%$ as a second- or thirdline therapy). Three patients achieved partial response and the disease control rate and PFS were $60 \%$ and 7.6 months respectively (57). Lastly, 11 patients received ICIs among 129 RET-rearranged NSCLC collected in 13 Chinese centers. Among 10 patients with evaluable response, disease control rate was $60 \%$, and ORR was $20 \%$. PFS was overall poor (3.8 months), but two patients had durable response of 10.4 and 11.5 months at data cut off. Interestingly, both patients harbored KIF5B-RET fusion and presented high PD-L1 expression (TPS >50\%) (49) (Table 1). Efficacy of combinations between immunotherapy and selective RET inhibitors have not been assessed yet. As far as we know, there are not ongoing clinical trials evaluating this treatment strategy.

\section{Emerging oncogene rearrangements}

Neurotrophic tyrosine kinase- (NTRK), neuregulin 1- (NRG1), and fibroblast growth factor receptor 3(FGFR3) fusions have been recently proposed as novel oncogene drivers for a small percentage of NSCLC (60-63). Selective TKIs targeting NTRK rearrangements, like entrectinib and larotrectinib, are currently being tested for several tumor types, (60) with both agents showing exciting activity also for NTRK positive NSCLC $(64,65)$. However, where clinical trials for NTRK inhibitors are not available, chemotherapy and/or immunotherapy remains the standard first line treatment options for NTRK positive NSCLC. Alongside c-MET amplification and exon 14 skipping mutation, KIF5B-MET, HLA-DRB1-MET, and MET-ATXN7L1 fusions have also been recently reported as oncogenic drivers in NSCLC (66-68). The clinical relevance of these rearrangements is still unclear, but their onset could be unrelated to other oncogene alterations and previous TKI treatments. Although novel TKIs are currently being tested for the different c-MET alterations, MET fusions have been excluded from the majority of these clinical trials (e.g., NCT02864992, NCT04077099, NCT03940703, NCT03911193).

\section{Pre-clinical background}

Comprehensive genomic profiling showed a higher TMB and PD-L1 expression for NTRK rearranged NSCLC compared to other oncogene addicted tumors harboring
EGFR mutations, ALK or ROS1 rearrangements (69). However, NTRK fusions in NSCLC often co-exist with STK11 mutations, which have been associated to poor immunotherapy response in KRAS mutant disease (70). A global, multicenter network of thoracic oncologists (6 countries, 13 institutions) identified 80 patients with pathologically confirmed NRG1 fusion-positive NSCLCs; when tested, PD-L1 was found negative in most of these tumors $(79 \%, 26 / 33)$ (71). Qin et al. evaluated TMB in FGFR rearranged NSCLC, showing relatively low levels (5.2 Mb), in the majority of tested adenocarcinomas, consistently with the results obtained for other oncogene addicted NSCLC. As expected, TMB was higher in squamous cell carcinomas (9.6 Mb) (63). c-MET activation induces PD-L1 expression regardless from JAK/STAT signaling pathway $(72,73)$. It was associated also to the upregulation of other immunosuppressive genes and transcripts involved in angiogenesis and cell proliferation (72). Moreover, Wang et al. demonstrated that the inhibition of c-MET decreased indoleamine-2,3-dioxygenase expression (74), which exerts immunosuppressive effects on T-cells and natural killer cells (75). These data suggest that MET activation could be involved in the tumors immune escape process, likely contributing to an immunosuppressive microenvironment (76). Although specific conclusions for MET fusions cannot be currently drawn, it is likely that the high PD-L1 expression and low-TMB level found in c-MET exon 14 skipping mutated NSCLC samples (77) may be likely applied also to other MET alterations, including oncogenic rearrangements.

\section{Clinical evidence}

Duruisseaux et al. observed no ORR among 6 NSCLC patients harboring NRG1 fusions who were treated with anti-PD-1 or anti-PD-L1 therapy (71). Although the high frequency of high PD-L1 expression, single agent ICIs activity in c-MET exon 14 skipping mutated NSCLC patients was very poor within different retrospective series $(26,77)$, while favorable outcomes to ICIs therapy have been observed in patients with high c-MET expression (78). No evidence is currently available regarding the clinical efficacy of ICIs in MET, NTRK, or FGFR3 rearranged NSCLCs.

\section{Conclusions}

The potential role of ICI therapy in NSCLC patients harboring oncogenic fusions represents an actual and 
controversial topic which requires further investigation in dedicated studies. Pre-clinical data revealed that ALK/ ROS1/RET rearranged NSCLC are mostly characterized by low TMB level, suggesting that high PD-L1 expression may just be an epiphenomenon of the oncogene pathway activity rather than a crucial mechanism of cancer immuneescape. Current available evidence (Table 1) overall showed that tumors harboring oncogene rearrangements likely do not represent the best candidate to single agent ICI therapy, with poor clinical responses to PD1/PD-L1 inhibitors reported across different retrospective studies including TKI pre-treated populations. The higher incidence of rare oncogene rearrangements may likely explain also the lower efficacy of first-line ICIs in never smoker patients with high PD-L1 expression (79), highlighting the issue of adequate molecular testing for upfront treatment selection. Despite a strong biological rationale and the high expectation regarding the association between TKI and immunotherapy, early clinical trials disappointing results discouraged to pursue this treatment strategy. Combination therapy with next-generation TKIs, which are usually associated to a lower toxicity, could still represents a viable option, worthy of investigation in dedicated trials. However, the specific impact of immunotherapy on already long-lasting TKI-related response might be difficult to prove and a serious evaluation of cost/effectiveness ratio, also in terms of financial toxicities, is mandatory. Considering the recent advent of immune-chemotherapy regimens in clinical practice, exploring the potential efficacy of this treatment strategy in specific subsets of NSCLC patients harboring oncogenic fusions represents a major topic for clinical research. Although the occurrence of ALK/ROS1 secondary mutations represent the main resistance mechanisms emerging under TKI therapy $(80,81)$, however, a significant subgroup of ALK/ROS1+ NSCLC patients treated with second/first-generation TKI, and most of those treated with third-generation TKI develop a non-oncogene driver dependent mechanism of resistance, being the best candidate for a combination approach (82). Furthermore, the recent evidence that lorlatinib activity after second-generation TKI resistance is strictly dependent from the presence of secondary ALK mutations (83) is leading to the development of genomicdriven therapeutic sequences in ALK/ROS1 positive NSCLC patients, ultimately favoring the investigation of alternative strategies in mutation-negative subgroups. The large-scale spreading of next generations sequencing panels will likely lead to an increased detection of rare oncogene rearrangement in NSCLC, with a potential correlation to both PD-L1 expression and ICIs' efficacy. Considering the low prevalence of oncogenic fusions in the overall NSCLC population, the design of prospective clinical studies in this subset of patients represents a real challenge for the academic community, but remains the best way to definitively assess whether there will be any place for ICIs therapy in the treatment algorithm of fusion driven NSCLC.

\section{Acknowledgments}

Funding: None.

\section{Footnote}

Provenance and Peer Review: This article was commissioned by the Guest Editors (Silvia Novello, Francesco Passiglia) for the series "Looking for Chimeras in NSCLC: Widen Therapeutic Options Targeting Oncogenic Fusions" published in Translational Lung Cancer Research. The article was sent for external peer review organized by the Guest Editors and the editorial office.

Reporting Checklist: The authors have completed the NARRATIVE REVIEW reporting checklist. Available at http://dx.doi.org/10.21037/tlcr-20-710

Peer Review File: Available at http://dx.doi.org/10.21037/ tlcr-20-710

Conflicts of Interest: All authors have completed the ICMJE uniform disclosure form (available at http:// dx.doi.org/10.21037/tlcr-20-710). The series "Looking for Chimeras in NSCLC: Widen Therapeutic Options Targeting Oncogenic Fusions" was commissioned by the editorial office without any funding or sponsorship. SN serves as an unpaid editorial board member of Translational Lung Cancer Research from Jul 2014 to Jul 2021. SN and FP served as the unpaid Guest Editors of the series. FP reports personal fees from MSD, Boehringer Ingelheim, and Astra Zeneca, outside the submitted work. SN reports personal fees from Eli Lilly, MSD, Roche, BMS, Takeda, Pfizer, Astra Zeneca, and Boehringer Ingelheim, outside the submitted work. PB reports personal fees from Astra Zeneca, MSD, BMS, and Roche, outside the submitted work. The other authors have no other conflicts of interest to declare. 
Ethical Statement: The authors are accountable for all aspects of the work in ensuring that questions related to the accuracy or integrity of any part of the work are appropriately investigated and resolved.

Open Access Statement: This is an Open Access article distributed in accordance with the Creative Commons Attribution-NonCommercial-NoDerivs 4.0 International License (CC BY-NC-ND 4.0), which permits the noncommercial replication and distribution of the article with the strict proviso that no changes or edits are made and the original work is properly cited (including links to both the formal publication through the relevant DOI and the license). See: https://creativecommons.org/licenses/by-nc-nd/4.0/.

\section{References}

1. ASCO. 2019 State of Lung Cancer Report Released. Accessed 17 May 2020.

2. Kris MG, Johnson BE, Berry LD, et al. Using multiplexed assays of oncogenic drivers in lung cancers to select targeted drugs. JAMA 2014;311:1998-2006.

3. Garrido G, Rabasa A, Garrido C, et al. Preclinical modeling of EGFR-specific antibody resistance: oncogenic and immune-associated escape mechanisms. Oncogene 2014;33:3129-39.

4. Lee CK, Man J, Lord S, et al. Checkpoint inhibitors in metastatic EGFR-mutated non-small cell lung cancer-a meta-analysis. J Thorac Oncol 2017;12:403-7.

5. Kowanetz M, Socinski MA, Zou W, et al. Abstract CT076: IMpower150: Efficacy of atezolizumab (atezo) plus bevacizumab (bev) and chemotherapy (chemo) in 1L metastatic nonsquamous NSCLC (mNSCLC) across key subgroups. Chicago: Proceedings: AACR Annual Meeting 2018, 2018:14-8.

6. Peters S, Camidge DR, Shaw AT, et al. Alectinib versus crizotinib in untreated ALK-positive non-small-cell lung cancer. N Engl J Med 2017;377:829-38.

7. Camidge DR, Kim HR, Ahn MJ, et al. Brigatinib versus crizotinib in ALK-positive non-small-cell lung cancer. N Engl J Med 2018;379:2027-39.

8. Solomon BJ, Besse B, Bauer TM, et al. Lorlatinib in patients with ALK-positive non-small-cell lung cancer: results from a global phase 2 study. Lancet Oncol 2018;19:1654-67.

9. Ota K, Azuma K, Kawahara A, et al. Induction of PDL1 Expression by the EML4-ALK Oncoprotein and Downstream Signaling Pathways in Non-Small Cell Lung
Cancer. Clin Cancer Res 2015;21:4014-21.

10. Garon EB, Rizvi NA, Hui R, et al. Pembrolizumab for the treatment of non-small-cell lung cancer. $\mathrm{N}$ Engl J Med 2015;372:2018-28.

11. Marzec M, Zhang Q, Goradia A, et al. Oncogenic kinase NPM/ALK induces through STAT3 expression of immunosuppressive protein CD274 (PD-L1, B7-H1). Proc Natl Acad Sci 2008;105:20852-7.

12. Koh J, Jang JY, Keam B, et al. EML4-ALK enhances programmed cell death-ligand 1 expression in pulmonary adenocarcinoma via hypoxia-inducible factor (HIF)- $1 \alpha$ and STAT3. Oncoimmunology 2015;5:e1108514.

13. Noman MZ, Desantis G, Janji B, et al. PD-L1 is a novel direct target of HIF-1 $\alpha$, and its blockade under hypoxia enhanced MDSC-mediated T cell activation. J Exp Med 2014;211:781-90.

14. Nouri K, Azad T, Lightbody E, et al. A kinome-wide screen using a NanoLuc LATS luminescent biosensor identifies ALK as a novel regulator of the Hippo pathway in tumorigenesis and immune evasion. FASEB J 2019;33:12487-99.

15. Roussel H, De Guillebon E, Biard L, et al. Composite biomarkers defined by multiparametric immunofluorescence analysis identify ALK-positive adenocarcinoma as a potential target for immunotherapy. Oncoimmunology 2017;6:e1286437.

16. Cha YJ, Shim HS. PD-L1 expression and CD8+ tumor-infiltrating lymphocytes are associated with ALK rearrangement and clinicopathological features in inflammatory myofibroblastic tumors. Oncotarget 2017;8:89465-74.

17. Spigel DR, Schrock AB, Fabrizio D, et al. Total mutation burden (TMB) in lung cancer (LC) and relationship with response to PD-1/PD-L1 targeted therapies. J Clin Oncol 2016;34:9017.

18. Liu SY, Dong ZY, Wu SP, et al. Clinical relevance of PD$\mathrm{L} 1$ expression and CD8+ T cells infiltration in patients with EGFR-mutated and ALK-rearranged lung cancer. Lung Cancer 2018;125:86-92.

19. Zhou P, Shaffer DR, Alvarez Arias DA, et al. In vivo discovery of immunotherapy targets in the tumour microenvironment. Nature 2014;506:52-7.

20. Borghaei H, Paz-Ares L, Horn L, et al. Nivolumab versus docetaxel in advanced nonsquamous non-small-cell lung cancer. N Engl J Med 2015;373:1627-39.

21. Herbst RS, Baas P, Kim DW, et al. Pembrolizumab versus docetaxel for previously treated, PD-L1-positive, advanced non-small-cell lung cancer (KEYNOTE-010): a 
randomised controlled trial. Lancet 2016;387:1540-50.

22. Reck M, Rodríguez-Abreu D, Robinson AG, et al. Updated analysis of KEYNOTE-024: pembrolizumab versus platinum-based chemotherapy for advanced nonsmall-cell lung cancer with PD-L1 tumor proportion score of 50\% or greater. J Clin Oncol 2019;37:537-46.

23. Garassino MC, Cho BC, Kim JH, et al. Durvalumab as third-line or later treatment for advanced non-small-cell lung cancer (ATLANTIC): an open-label, single-arm, phase 2 study. Lancet Oncol 2018;19:521-36.

24. Fehrenbacher L, von Pawel J, Park K, et al. Updated efficacy analysis including secondary population results for OAK: a randomized phase III study of atezolizumab versus docetaxel in patients with previously treated advanced non-small cell lung cancer. J Thorac Oncol 2018;13:1156-70.

25. Socinski MA, Jotte RM, Cappuzzo F, et al. Atezolizumab for first-line treatment of metastatic nonsquamous NSCLC. N Engl J Med 2018;378:2288-301.

26. Mazieres J, Drilon A, Lusque A, et al. Immune checkpoint inhibitors for patients with advanced lung cancer and oncogenic driver alterations: results from the IMMUNOTARGET registry. Ann Oncol 2019;30:1321-8.

27. Ng TL, Liu Y, Dimou A, et al. Predictive value of oncogenic driver subtype, programmed death-1 ligand (PD-L1) score, and smoking status on the efficacy of PD-1/PD-L1 inhibitors in patients with oncogene-driven non-small cell lung cancer. Cancer 2019;125:1038-49.

28. Gainor JF, Shaw AT, Sequist LV, et al. EGFR mutations and ALK rearrangements are associated with low response rates to $\mathrm{PD}-1$ pathway blockade in non-small cell lung cancer: a retrospective analysis. Clin Cancer Res 2016;22:4585-93.

29. Heo JY, Park C, Keam B, et al. The efficacy of immune checkpoint inhibitors in anaplastic lymphoma kinasepositive non-small cell lung cancer. Thorac Cancer 2019;10:2117-23.

30. Bylicki O, Guisier F, Monnet I, et al. Efficacy and safety of programmed cell-death-protein-1 and its ligand inhibitors in pretreated patients with epidermal growthfactor receptor-mutated or anaplastic lymphoma kinasetranslocated lung adenocarcinoma. Medicine (Baltimore) 2020;99:e18726.

31. Chalmers AW, Patel S, Boucher K, et al. Phase I trial of targeted EGFR or ALK therapy with ipilimumab in metastatic NSCLC with long-term follow-up. Target Oncol 2019;14:417-21.

32. Brahmer J, Reckamp KL, Baas P, et al. Nivolumab versus docetaxel in advanced squamous-cell non-small-cell lung cancer. N Engl J Med 2015;373:123-35.

33. Solomon BJ, Mok T, Kim DW, et al. First-line crizotinib versus chemotherapy in ALK-positive lung cancer. N Engl J Med 2014;371:2167-77.

34. Spigel DR, Reynolds C, Waterhouse D, et al. Phase $1 / 2$ study of the safety and tolerability of nivolumab plus crizotinib for the first-line treatment of anaplastic lymphoma kinase translocation - positive advanced nonsmall cell lung cancer (CheckMate 370). J Thorac Oncol 2018;13:682-8.

35. Patel SP, Pakkala S, Pennell NA, et al. Phase Ib study of crizotinib plus pembrolizumab in patients with previously untreated advanced non-small cell lung cancer with ALK translocation. Oncologist 2020;25:562-e1012.

36. Felip E, de Braud FG, Maur M, et al. Ceritinib plus nivolumab in patients with advanced ALK-rearranged non-small cell lung cancer: results of an openlabel, multicenter, phase 1B study. J Thorac Oncol 2020;15:392-403.

37. Soria JC, Tan DSW, Chiari R, et al. First-line ceritinib versus platinum-based chemotherapy in advanced ALKrearranged non-small-cell lung cancer (ASCEND-4): a randomised, open-label, phase 3 study. Lancet 2017;389:917-29.

38. Shaw AT, Kim TM, Crinò L, et al. Ceritinib versus chemotherapy in patients with ALK-rearranged nonsmall-cell lung cancer previously given chemotherapy and crizotinib (ASCEND-5): a randomised, controlled, openlabel, phase 3 trial. Lancet Oncol 2017;18:874-86.

39. Kim DW, Gadgeel SM, Gettinger SN, et al. Safety and clinical activity results from a phase Ib study of alectinib plus atezolizumab in ALK+ advanced NSCLC (aNSCLC). J Clin Oncol 2018;36:abstr 9009.

40. Camidge DR, Peters S, Mok T, et al. Updated efficacy and safety data from the global phase III ALEX study of alectinib (ALC) vs crizotinib (CZ) in untreated advanced ALK+ NSCLC. J Clin Oncol 2018;36:abstr 9043.

41. Shaw AT, Lee SH, Ramalingam SS, et al. Avelumab (anti-PD-L1) in combination with crizotinib or lorlatinib in patients with previously treated advanced NSCLC: Phase $1 \mathrm{~b}$ results from JAVELIN Lung 101. J Clin Oncol 2018;36:abstr 9008.

42. Shaw AT, Riely GJ, Bang YJ, et al. Crizotinib in ROS1rearranged advanced non-small-cell lung cancer (NSCLC): updated results, including overall survival, from PROFILE 1001. Ann Oncol 2019;30:1121-6.

43. Shaw AT, Solomon BJ, Chiari R, et al. Lorlatinib in 
advanced ROS1-positive non-small-cell lung cancer: a multicentre, open-label, single-arm, phase 1-2 trial. Lancet Oncol 2019;20:1691-701.

44. Drilon A, Siena S, Dziadziuszko R, et al. Entrectinib in ROS1 fusion-positive non-small-cell lung cancer: integrated analysis of three phase 1-2 trials. Lancet Oncol 2020;21:261-70.

45. Cho BC, Drilon AE, Doebele RC, et al. Safety and preliminary clinical activity of repotrectinib in patients with advanced ROS1 fusion-positive non-small cell lung cancer (TRIDENT-1 study). J Clin Oncol 2019;37:abstr 9011.

46. Katayama R, Gong B, Togashi N, et al. The newgeneration selective ROS1/NTRK inhibitor DS-6051b overcomes crizotinib resistant ROS1-G2032R mutation in preclinical models. Nat Commun 2019;10:3604.

47. Rangachari D, VanderLaan PA, Shea M, et al. Correlation between classic driver oncogene mutations in EGFR, ALK, or ROS1 and 22C3-PD-L1 $250 \%$ expression in lung adenocarcinoma. J Thorac Oncol 2017;12:878-83.

48. Karatrasoglou EA, Chatziandreou I, Sakellariou S, et al. Association between PD-L1 expression and driver gene mutations in non-small cell lung cancer patients: correlation with clinical data. Virchows Arch 2020;477:207-17.

49. Lu C, Dong XR, Zhao J, et al. Association of genetic and immuno-characteristics with clinical outcomes in patients with RET-rearranged non-small cell lung cancer: a retrospective multicenter study. J Hematol Oncol 2020;13:37.

50. Park S, Ahn BC, Lim SW, et al. Characteristics and outcome of ROS1-positive non-small cell lung cancer patients in routine clinical practice. J Thorac Oncol 2018;13:1373-82.

51. Gainor JF, Lee DH, Curigliano G, et al. Clinical activity and tolerability of BLU-667, a highly potent and selective RET inhibitor, in patients (pts) with advanced RETfusion+ non-small cell lung cancer (NSCLC). J Clin Oncol 2019;37:abstr 9008.

52. Drilon A, Oxnard G, Wirth L, et al. PL02.08 registrational results of LIBRETTO-001: a phase 1/2 trial of LOXO292 in patients with RET fusion-positive lung cancers. J Thorac Oncol 2019;14:S6-7.

53. Schuringa JJ, Wojtachnio K, Hagens W, et al. MEN2ARET-induced cellular transformation by activation of STAT3. Oncogene 2001;20:5350-8.

54. Castellone MD, Melillo RM. RET-mediated modulation of tumor microenvironment and immune response in multiple endocrine neoplasia type 2 (MEN2). Endocr Relat Cancer 2018;25:T105-19.

55. Song Z, Yu X, Cheng G, et al. Programmed death-ligand 1 expression associated with molecular characteristics in surgically resected lung adenocarcinoma. J Transl Med 2016;14:188.

56. Offin M, Guo R, Wu SL, et al. Immunophenotype and response to immunotherapy of RET-rearranged lung cancers. JCO Precis Oncol 2019;3:10.1200/PO.18.00386.

57. Guisier F, Dubos-Arvis C, Viñas F, et al. Efficacy and safety of anti-PD-1 immunotherapy in patients with advanced NSCLC with BRAF, HER2, or MET mutations or RET translocation: GFPC 01-2018. J Thorac Oncol 2020;15:628-36.

58. Sarfaty M, Moore A, Neiman V, et al. RET fusion lung carcinoma: response to therapy and clinical features in a case series of 14 patients. Clin Lung Cancer 2017;18:e223-32.

59. Baglivo S, Ludovini V, Moretti R, et al. RET rearrangement as a predictor of unresponsiveness to immunotherapy in non-small cell lung cancer: report of two cases with review of the literature. Oncol Ther 2020;8:333-9.

60. Ricciuti B, Brambilla M, Metro G, et al. Targeting NTRK fusion in non-small cell lung cancer: rationale and clinical evidence. Med Oncol 2017;34:105.

61. Ke H, Shen W, Hu A, et al. Distribution of NRG1 gene fusions in a large population of Chinese patients with NSCLC. J Thorac Oncol 2019;14:e263-6.

62. Jonna S, Feldman RA, Swensen J, et al. Detection of NRG1 gene fusions in solid tumors. Clin Cancer Res 2019;25:4966-72.

63. Qin A, Johnson A, Ross JS, et al. Detection of known and novel FGFR fusions in non-small cell lung cancer by comprehensive genomic profiling. J Thorac Oncol 2019;14:54-62.

64. Doebele RC, Drilon A, Paz-Ares L, et al. Entrectinib in patients with advanced or metastatic NTRK fusionpositive solid tumours: integrated analysis of three phase 1-2 trials. Lancet Oncol 2020;21:271-82.

65. Hong DS, DuBois SG, Kummar S, et al. Larotrectinib in patients with TRK fusion-positive solid tumours: a pooled analysis of three phase $1 / 2$ clinical trials. Lancet Oncol 2020;21:531-40.

66. Wang $\mathrm{W}, \mathrm{Xu} \mathrm{C}$, Chen $\mathrm{Y}$, et al. MET gene fusions in nonsmall cell lung cancer (NSCLC) in the Chinese population: a multicenter study. J Clin Oncol 2018;36:e13539.

67. Davies KD, Ng TL, Estrada-Bernal A, et al. Dramatic 
response to crizotinib in a patient with lung cancer positive for an HLA-DRB1-MET gene fusion. JCO Precis Oncol 2017. doi: 10.1200/PO.17.00117.

68. Gow CH, Liu YN, Li HY, et al. Oncogenic function of a KIF5B-MET fusion variant in non-small cell lung cancer. Neoplasia 2018;20:838-47.

69. Ou SHI, Sokol ES, Trabucco SE, et al. NTRK1-3 genomic fusions in non-small cell lung cancer (NSCLC) determined by comprehensive genomic profiling. Ann Oncol 2019;30:v638.

70. Skoulidis F, Goldberg ME, Greenawalt DM, et al. STK11/LKB1 mutations and PD-1 inhibitor resistance in KRAS-mutant lung adenocarcinoma. Cancer Discov 2018;8:822-35.

71. Duruisseaux M, Drilon A, Han JY, et al. NRG1 fusionpositive lung cancers: clinicopathologic profile and treatment outcomes from a global multicenter registry. Eur Respir J 2019;54:PA3666.

72. Saigi M, Alburquerque-Bejar JJ, Mc Leer-Florin A, et al. MET-oncogenic and JAK2-inactivating alterations are independent factors that affect regulation of PD-L1 expression in lung cancer. Clin Cancer Res 2018;24:4579-87.

73. Albitar M, Sudarsanam S, Ma W, et al. Correlation of MET gene amplification and TP53 mutation with PDL1 expression in non-small cell lung cancer. Oncotarget 2018;9:13682-93.

74. Wang D, Saga Y, Sato N, et al. The hepatocyte growth factor antagonist NK4 inhibits indoleamine-2,3dioxygenase expression via the c-Met-phosphatidylinositol 3-kinase-AKT signaling pathway. Int J Oncol 2016;48:2303-9.

75. Frumento G, Rotondo R, Tonetti M, et al. Tryptophanderived catabolites are responsible for inhibition of $\mathrm{t}$ and natural killer cell proliferation induced by indoleamine 2,3-dioxygenase. J Exp Med 2002;196:459-68.

76. Titmarsh HF, O'Connor R, Dhaliwal K, et al. The emerging role of the c-MET-HGF axis in non-small lung cancer tumor immunology and immunotherapy. Front Oncol 2020;10:54.

77. Sabari JK, Leonardi GC, Shu CA, et al. PD-L1 expression, tumor mutational burden, and response to immunotherapy in patients with MET exon 14 altered lung cancers. Ann Oncol 2018;29:2085-91.

78. Reis H, Metzenmacher M, Goetz M, et al. MET expression in advanced non-small-cell lung cancer: effect on clinical outcomes of chemotherapy, targeted therapy, and immunotherapy. Clin Lung Cancer 2018;19:e441-63.

79. Gainor JF, Rizvi H, Jimenez Aguilar E, et al. Clinical activity of programmed cell death 1 (PD-1) blockade in never, light, and heavy smokers with non-small-cell lung cancer and PD-L1 expression $>50 \%$. Ann Oncol 2020;31:404-11.

80. Gainor JF, Dardaei L, Yoda S, et al. Molecular mechanisms of resistance to first- and second-generation ALK inhibitors in ALK-rearranged lung cancer. Cancer Discov 2016;6:1118-33.

81. Yoda S, Lin JJ, Lawrence MS, et al. Sequential ALK inhibitors can select for lorlatinib-resistant compound ALK mutations in ALK-positive lung cancer. Cancer Discov 2018;8:714-29.

82. Lin JJ, Zhu VW, Yoda S, et al. Impact of EML4-ALK variant on resistance mechanisms and clinical outcomes in ALK-positive lung cancer. J Clin Oncol 2018;36:1199-206.

83. Shaw AT, Solomon BJ, Besse B, et al. ALK resistance mutations and efficacy of lorlatinib in advanced anaplastic lymphoma kinase-positive non-small-cell lung cancer. J Clin Oncol 2019;37:1370-9.
Cite this article as: Leone G, Passiglia F, Bironzo P, Bertaglia V, Novello S. Is there any place for immune-checkpoint inhibitors in the treatment algorithm of fusion-driven non-small cell lung cancer?-a literature review. Transl Lung Cancer Res 2020;9(6):2674-2685. doi: 10.21037/tlcr-20-710 